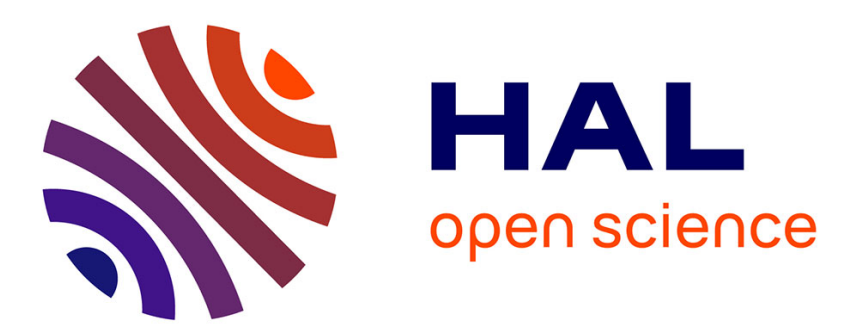

\title{
Theoretical search for solutions to minimize negative influence of segregation in mixing of particulate solids
}

\author{
Vadim Mizonov, Henri Berthiaux, Cendrine Gatumel
}

\section{To cite this version:}

Vadim Mizonov, Henri Berthiaux, Cendrine Gatumel. Theoretical search for solutions to minimize negative influence of segregation in mixing of particulate solids. Particuology , 2016, 25, p. 36-41. 10.1016/j.partic.2015.05.002 . hal-01599985

\section{HAL Id: hal-01599985 \\ https://hal.science/hal-01599985}

Submitted on 7 Nov 2019

HAL is a multi-disciplinary open access archive for the deposit and dissemination of scientific research documents, whether they are published or not. The documents may come from teaching and research institutions in France or abroad, or from public or private research centers.
L'archive ouverte pluridisciplinaire HAL, est destinée au dépôt et à la diffusion de documents scientifiques de niveau recherche, publiés ou non, émanant des établissements d'enseignement et de recherche français ou étrangers, des laboratoires publics ou privés. 


\title{
Theoretical search for solutions to minimize negative influence of segregation in mixing of particulate solids
}

\author{
Vadim Mizonov $^{\mathrm{a}, *}$, Henri Berthiaux ${ }^{\mathrm{b}}$, Cendrine Gatumel $^{\mathrm{b}}$ \\ a Department of Applied Mathematics, Ivanovo State Power Engineering University, Rabfakovskaya 34, 153003 Ivanovo, Russia \\ ${ }^{\mathrm{b}}$ Centre RAPSODEE, UMR CNRS 5302, Ecole des Mines d'Albi-Carmaux, Campus Jarlard, route de Teillet, 81000 Albi, France
}

\section{Keywords:}

Mixing

Segregation

Mixture quality

Markov chain

Optimization

\begin{abstract}
A B S T R A C T
The objective of this study is to examine several optimization problems in the batch mixing of segregating particulate solids that can be set up and solved using Markov chain models. To improve the adequacy of such models and exclude some physical contradictions that arise in the linear form, a non-linear Markov chain model for the mixing of segregating components is proposed. Optimal solutions are obtained by controlling the particle flow outside the mixing operating volume while the components are being loaded, modifying particle circulation inside the mixing zone during the process, and by structuring the load in the mixing zone. Solutions are found that not only reduce the negative influence of segregation, but also exclude it altogether. The gain resulting from optimization grows with the rate of segregation. The optimal solutions presented here can be used to improve the design of mixers.
\end{abstract}

\section{Introduction}

The mixing of solids is a complex process involving particles migrating inside the operating volume of a mixer. A feature of this process is that the components to be mixed often have a tendency to segregate within each other. The segregation occurs because of differences in the physical properties of the components, such as particle size, density, shape, etc. The action of gravity is always present in mixing, but affects different particles in different ways, leading to segregation. It is practically impossible to achieve a homogeneous mixture of segregating components, at least in industrial-scale apparatus. However, it is possible to decrease the negative influence of segregation by controlling the flow of particles in the components to be mixed.

Modeling the process mathematically enables a better understanding, which in turn allows us to search for methods of improvement. Different approaches have been used to model the mixing of solids. In early studies of the mixing development, analytical models based on the continuous convection-diffusion equation were widely used (for example, Danckwerts (1953) and Sommer (1996)). Despite the fact that it is still sometimes used, the analytical results obtained with this approach are mostly of academic interest, because unrealistic assumptions must be imposed

\footnotetext{
* Corresponding author. Tel.: +7 910 9948858; fax: +7 4932385701 E-mail address: mizonov46@mail.ru (V. Mizonov).
}

to obtain analytical solutions, and important physical features of the process appear to be missed. Iddir, Arastoopour, and Hrenya (2005) extended the kinetic theory to granular mixtures of different mechanical properties (size, density, and/or restitution coefficient), where each particle group was considered as a separate phase with different average velocity and granular energy. This model was applied to the simple shear flow of binary and ternary mixtures of particles. However, their paper did not consider optimization problems.

Bridgwater $(2010,2012)$ emphasized that the design and operation of the mixing process are very difficult, being largely based on judgments rather than science. The next stage of development is to build on emerging knowledge and methods so that the basics for design can be formulated. Design will then become predictable, with operation effectively controlling performance. Thus, the goal should be to take the designer through a sequence of steps to the most appropriate mixer size, configuration, and operating conditions for a given process. The author sees the discrete element method (DEM) simulation as an effective tool for this problem. However, this method is very time consuming, which presents a serious problem when it is necessary to calculate and compare a lot of variants in the process regime and internal configuration of a mixer.

Thus, despite the fact that many approaches have been used to model the mixing process, the problem of theoretical optimization is in the early stages of development. At this stage, it is important to choose an appropriate mathematical tool that allows the 


\begin{tabular}{|ll|}
\hline \multicolumn{2}{|c|}{ Nomenclature } \\
$D$ & dispersion coefficient, $\mathrm{m}^{2} / \mathrm{s}$ \\
$d$ & probability of diffusion transition \\
$k$ & transition number \\
$K$ & period of segregating component loading \\
$m$ & number of cells for segregating component load \\
$n$ & number of cells in a chain \\
$\mathbf{P}, P_{i j}$ & matrix of transition probabilities and its entries \\
$\mathbf{S}, S_{j}$ & state vector and its entries \\
$\mathbf{S m}$ & feed matrix \\
$V$ & rate of segregation, m/s \\
$v$ & probability of segregation transition \\
& \\
$G r e e k$ symbols \\
$\sigma$ & standard deviation \\
$\Delta t$ & transition duration, $\mathrm{s}$ \\
$\Delta x$ & height of a cell, $\mathrm{m}$ \\
\hline
\end{tabular}

optimization problems to be clearly defined and attains optimal solutions that are not too far from practical realization.

According to the authors' viewpoint, the theory of Markov chains is such a tool. This theory is naturally related to the process of mixing, because both concern the evolution of the state of a stochastic system. The basic idea of the Markov chain approach is to separate the operating volume of the mixer into small but finite zones (cells), and then observe the evolution of the key component concentrations in these zones at discrete points in time. The application of this approach to describe the state of a mixture after passing through a static mixer was reported by Wang and Fan (1976). However, in their work, the evolution of process parameters and the physical features of the mixing zone were not described. Their model, which only allows transitions to neighboring cells, was further developed by Wang and Fan (1977) and Fan, Lai, Akao, Shinoda, and Yoshizawa (1978).

The general strategy of applying the theory of Markov chains to model different processes in powder technology was described in our previous papers (Ammarcha et al., 2013; Berthiaux \& Mizonov, 2004; Berthiaux, Marikh, Mizonov, Ponomarev, \& Barantzeva, 2004; Berthiaux, Mizonov, \& Zhukov, 2005; Marikh, Berthiaux, Mizonov, Barantseva, \& Ponomarev, 2006). However, these reports generally use the theory to describe the process, rarely to optimize it. An attempt to use Markov chains to optimize the mixing process via the optimal control of segregating component flows is presented below.

\section{A non-linear chain model of batch mixing}

Detailed studies of the physical bases of the Markov chain models and the limits of their applicability were given by Berthiaux and Mizonov (2004) and Berthiaux et al. (2005). It is necessary to state the following concerning segregation. Since the objective of this study is a binary mixture of particulate solids, the downward segregation of a key component always goes together with the upward segregation of a basic component, in accordance with the continuity equation. In each cell, the relative content of the basic component is equal to one minus the relative content of the key component. Thus, to estimate the mixture quality, it is enough to know how the key component is distributed over the operating volume of a mixer. Namely, this distribution and its characteristics will be the objective function of optimization.

To set up optimization problems on the basis of more or less realistic models of the mixing process, let us examine the common linear Markov chain model, and identify some contradictions.

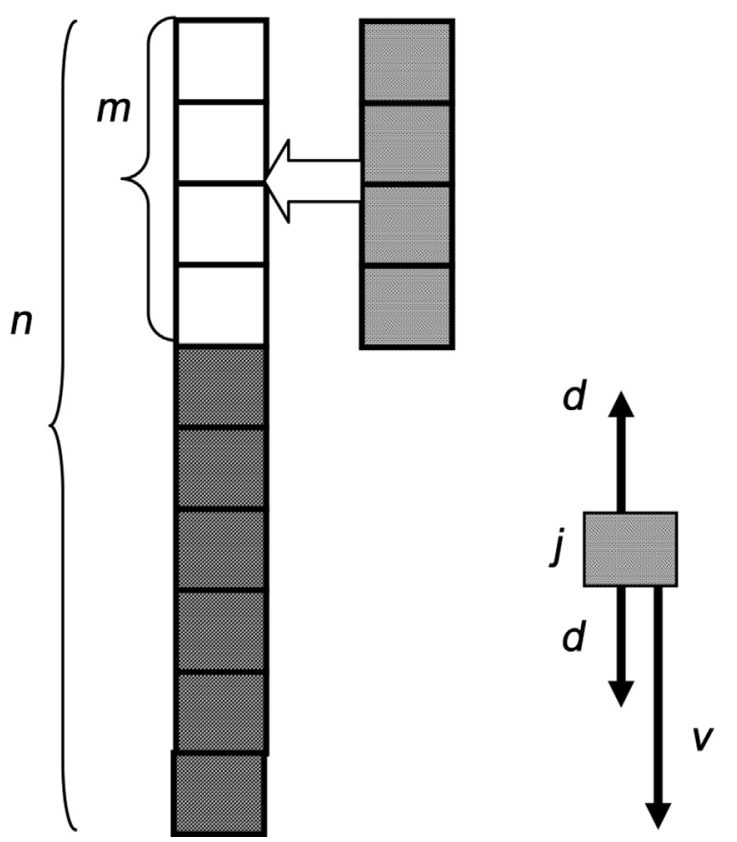

Fig. 1. Scheme of the cell model and structure of transitions from the $j$-th cell.

Suppose that a batch mixing zone is presented as a one-dimensional array of $n$ perfectly mixed cells, as shown in Fig. 1.

The key component distribution over the cells can be described by the column state vector $\mathbf{S}=\left\{S_{j}\right\}$ of size $n \times 1$. Assume that we can observe the state of the process at discrete moments of time $t_{k}=(k-1) \Delta t$, where $\Delta t$ is the transition duration and $k$ is the transition number, which can be interpreted as the discrete analog of time. In this case, the evolution of the key component state (i.e., mixing kinetics) can be described by the recurrent matrix equation

$\mathbf{S}^{k+1}=\mathbf{P S}^{k}$,

where $\mathbf{P}$ is the matrix of transition probabilities that distributes $\mathbf{S}$ over the cells at each time step, or transition. The $j$-th column of $\mathbf{P}$ belongs to the $j$-th cell, and contains the probabilities of transitioning to the neighboring cells, as shown in Fig. 1. These probabilities have a symmetrical part $d$ that is related to pure quasi-diffusion, which always leads to a flattening of the distribution, and a nonsymmetrical part $v$ that is related to segregation, which leads to non-homogeneity. The values of $d$ and $v$ can be calculated as: $d=D \Delta t / \Delta x^{2}, v=V \Delta t / \Delta x$, where $D$ is the dispersion coefficient, $V$ is the rate of segregation, and $\Delta x$ is the cell height. Thus, the matrix $\mathbf{P}$ has the following form

$\mathbf{P}=\left[\begin{array}{ccccc}1-d-v & d & 0 & 0 & \ldots \\ d+v & 1-2 d-v & d & 0 & \ldots \\ 0 & d+v & 1-2 d-v & d & \ldots \\ 0 & 0 & d+v & \ldots & \ldots \\ \ldots & \ldots & \ldots & \ldots & \ldots\end{array}\right]$.

Usually, $V$ (or $v$ ) is held constant during the process, and the model described by Eq. (1) is linear. However, this is a very rough assumption that can lead to both quantitative errors and qualitative contradictions. Indeed, a segregating component cannot segregate inside itself, and, if the $(j+1)$-th cell is filled with this component (i.e., contains $S_{\max }$ of it), the value of $v$ should be equal to zero. On the contrary, if the $(j+1)$-th cell does not contain any of the segregating component, the value of $v$ should be maximal. It is natural to 

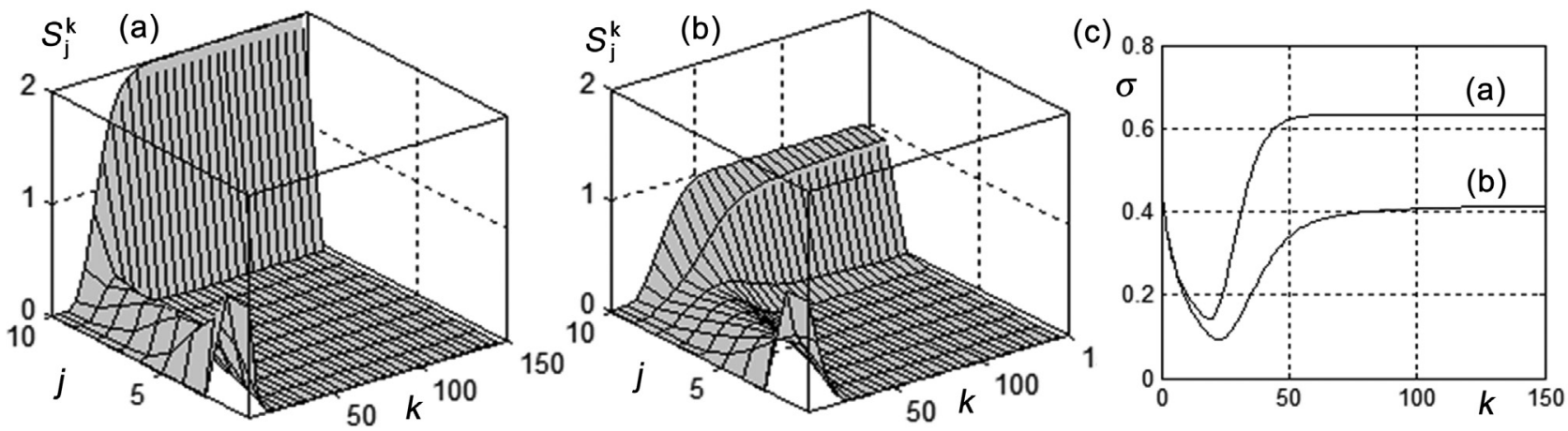

Fig. 2. Evolution of the key component distribution due to linear (a) and non-linear (b) cell model and mixing kinetics for both the models (c) at $d=0$ and $v=0.3$.

introduce the assumption that the dependence between $v$ and $S_{j+1}^{k}$ is linear:

$v_{j}^{k}=v_{0}\left(1-\frac{S_{j+1}^{k}}{S_{\max }}\right)$.

Thus, the model becomes non-linear, because the matrix $\mathbf{P}$ depends on the current state vector $\mathbf{S}^{k}$ and varies at each time transition. Under this assumption, the matrix $\mathbf{P}$ takes the form

$\mathbf{P}\left(\mathbf{S}^{k}\right)=\left[\begin{array}{ccccc}1-d-v\left(S_{2}^{k}\right) & d & 0 & 0 & \ldots \\ d+v\left(S_{2}^{k}\right) & 1-2 d-v\left(S_{3}^{k}\right) & d & 0 & \ldots \\ 0 & d+v\left(S_{3}^{k}\right) & 1-2 d-v\left(S_{4}^{k}\right) & d & \ldots \\ 0 & 0 & d+v\left(S_{4}^{k}\right) & \ldots & \ldots \\ \ldots & \ldots & \ldots & \ldots & \ldots\end{array}\right]$.

A comparison of the process descriptions given by the linear and non-linear models is shown in Fig. 2. At the initial point in time, the two upper cells are filled with the segregating component (hereinafter, it is supposed that $S_{\max }=1$ ). As a practical example, the segregating component could be fine sand on top of coarse particles. The input of energy and progress of time cause the fine particles to trickle through the coarse particles and accumulate at the bottom of the vessel as a result of gravity.

The linear model (Fig. 2(a), $v=0.3=$ const.) gives an asymptotic distribution, in which these two upper portions appear in one and the same bottom cell, which is impossible. The asymptotic state of the mixture becomes more non-homogeneous than the initial one. On the contrary, the asymptotic distribution in the non-linear model gives two lower cells filled with the component, which is realistic. Fig. 2(c) shows the evolution of the non-homogeneity of the mixture in terms of the standard deviation of the key component content distribution, $\sigma$. In the linear model, $\sigma$ reaches a minimum earlier, but this is greater than the minimum attained by the non-linear model, i.e., this model reaches a higher maximum mixing quality.

\section{Formulation of the optimization problem}

The technological objective of mixing solids is to obtain a mixture in which a key component is distributed over the mixture volume as homogeneously as possible, or meets the technological requirements of mixture non-homogeneity. If the non-homogeneity is characterized by the standard deviation of a key component distribution $\sigma$, this condition can be formulated as $\sigma \rightarrow \sigma_{\min }$, or $\sigma \leq \sigma_{c}$. A common way to begin the mixing process is to place a segregating key component at the top of a mixing zone (as shown in Fig. 1), and to run the mixer, i.e., to agitate the mixture by mechanical action (vibration, blades, etc.). If the agitation parameters are given (i.e., the values of $d$ and $v$ are defined), the only way to optimize the process is to find the optimal mixing time (see, for example, Fig. 2(c)) at which $\sigma=\sigma_{\min }$. However, what can be done if $\sigma_{\min }$ is greater than $\sigma_{c}$ and does not meet the technological requirements? According to the kinetic Eq. (1), there are two possibilities: control the load of a key component into a mixer (outside the matrix), or control the matrix of transition probabilities at the same values of $v$ and $d$ (inside the matrix).

\section{Optimal control of load flow}

\section{Time-distributed load of a key component}

Suppose that the total volume of a key component is such that it occupies $m$ upper cells of total $n$ cells (see Fig. 1 ). At the point at which the key component is loaded into the mixer, the initial state vector of its distribution is

$S_{j}^{0}=\left\{\begin{array}{ll}1, & 1 \leq j \leq m \\ 0, & m<j \leq n\end{array}\right.$,

and its further transformation, in terms of segregating the upper particles downward over the total time, is described by Eq. (1). It is obvious that the negative influence of segregation will be mitigated if the segregation time decreases with growing level of the particles. This can be achieved by means of the time-distributed loading of the segregating component into the mixer, as shown in Fig. 3.

In this case, we have something very similar to continuous mixing that goes on until the key component is fully loaded, after which we deal with pure batch mixing. Such a process can be called semi-batch (or semi-continuous) mixing. The kinetics of continuous mixing can also be described by the recurrent matrix equation

$\mathbf{S}^{k+1}=\mathbf{P S}^{k}+\mathbf{S}_{f}^{k}$,

where $\boldsymbol{S}_{\mathbf{f}}^{k}$ is the feed vector that describes the amount of particles added to the cells of the chain at each time transition.

Let us suppose that the cells $m, m-1, m-2, \ldots$ are filled with the segregating key component after $K$ time transitions. In this case, the value of $1 / K$ is the analog of the feed flow rate of a feeder that fills the mixer during $m K$ time transitions. The feed vector for such 


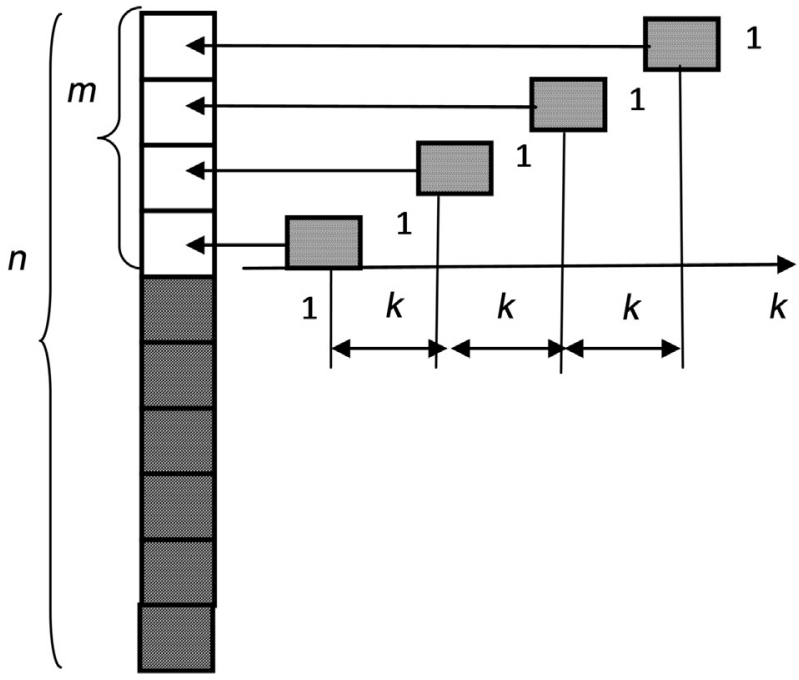

Fig. 3. Scheme of the cell model with time-distributed load of segregating component.

a process can be extracted from the feed matrix. An example for $m=3$ and $K=3$ is shown below

$\mathbf{S m}_{f}^{k}=\left[\begin{array}{cccccccccc}0 & 0 & 0 & 0 & 0 & 0 & 1 & 0 & 0 & \ldots \\ 0 & 0 & 0 & 1 & 0 & 0 & 0 & 0 & 0 & \ldots \\ 1 & 0 & 0 & 0 & 0 & 0 & 0 & 0 & 0 & \ldots \\ 0 & 0 & 0 & 0 & 0 & 0 & 0 & 0 & 0 & \ldots \\ \ldots & \ldots & \ldots & \ldots & \ldots & \ldots & \ldots & \ldots & \ldots & \ldots\end{array}\right]$.

The $n \times 1$ matrix of transition probabilities must be written for the whole chain. However, the cells that are not involved in the process at the current time transition must be locked to prevent particles entering. This can be done as follows

$\mathbf{P}=\left[\begin{array}{ccccc}1-d-v_{1}^{k} & e_{2}^{k} d & 0 & 0 & \ldots \\ d+v_{1}^{k} & 1-d-e_{2}^{k} d-v_{2}^{k} & e_{3}^{k} d & 0 & \ldots \\ 0 & d+v_{2}^{k} & 1-d-e_{3}^{k} d-v_{3}^{k} & e_{4}^{k} d & \ldots \\ 0 & 0 & d+v_{3}^{k} & \ldots & \ldots \\ \ldots & \ldots & \ldots & \ldots & \ldots\end{array}\right]$,

where the coefficient $e_{j}^{k}$ blocks the upper part of the chain if it is equal to zero, and opens it up when equal to one. This coefficient can also be extracted from the following matrix (example for $m=3$ and $K=3$ )

$e=\left[\begin{array}{cccccccccc}1 & 1 & 1 & 1 & 1 & 1 & 1 & 1 & 1 & \ldots \\ 0 & 0 & 0 & 0 & 0 & 0 & 1 & 1 & 1 & \ldots \\ 0 & 0 & 0 & 1 & 1 & 1 & 1 & 1 & 1 & \ldots \\ 1 & 1 & 1 & 1 & 1 & 1 & 1 & 1 & 1 & \ldots \\ \ldots & \ldots & \ldots & \ldots & \ldots & \ldots & \ldots & \ldots & \ldots & \ldots\end{array}\right]$.

It is easy to build algorithms to generate these matrices for any $n, m$, and $K$.

An example showing the evolution of mixture non-homogeneity for different variants of key component loading at $d=0.1$ and $v_{0}=0.3$ is illustrated in Fig. 4. Each curve begins at the point corresponding to complete loading of the key component.

The bold curve shows the evolution for one-time loading, the thin ones represent time-distributed loading with various periods $K$. It is obvious that the thin curves should only be examined after the number of transitions corresponding to complete loading of the

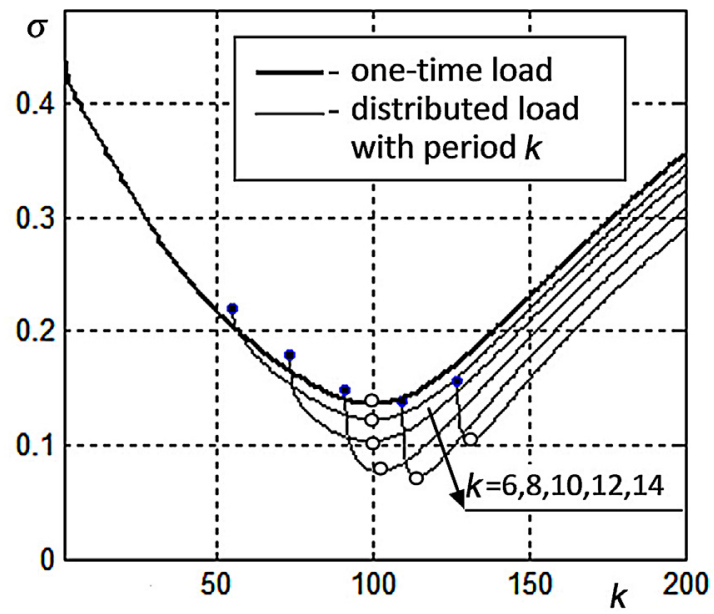

Fig. 4. Evolution of mixture non-homogeneity for different variants of key component loading at $d=0.1$ and $v=0.3$.

key component (the left edge of the curves marked by dark circles). There clearly exists an optimal value of $K=12$, which gives a value of $\sigma_{\min }$ that is almost half the magnitude of that for one-time loading.

Fig. 5 shows the generalization of numerical results for different values of $d$ and $v$. Each point of the surface for the time-distributed load corresponds to the optimal value of $K$ found in the numerical experiments. The gain resulting from optimization increases with the magnitude of $v$, and decreases with a rise in $d$. These findings are obvious from a physical perspective. It is interesting that the influence of $d$ and $v$ on $\sigma_{\min }$ in optimized regimes is much less than in the non-optimized ones. However, such phenomena are often found in optimization problems when the influence of process parameters is "washed out" after the optimization.

\section{Structured load}

If a segregating component is loaded into a mixer as several layers over its height, it is obvious that $\sigma_{\min }$ can be reached faster, and will be smaller, in comparison to loading the component at the very top of the mixer. However, loading the segregating component layer by layer increases the duration of the loading operation, and it is important to estimate the gain of this loading method in terms of mixing time and mixture quality. To examine this, it is sufficient to run the procedure given by Eq. (1) with different initial state vectors. An example of this operation is shown in Fig. 6, where the mixing kinetics for two initial state vectors are shown in the graph

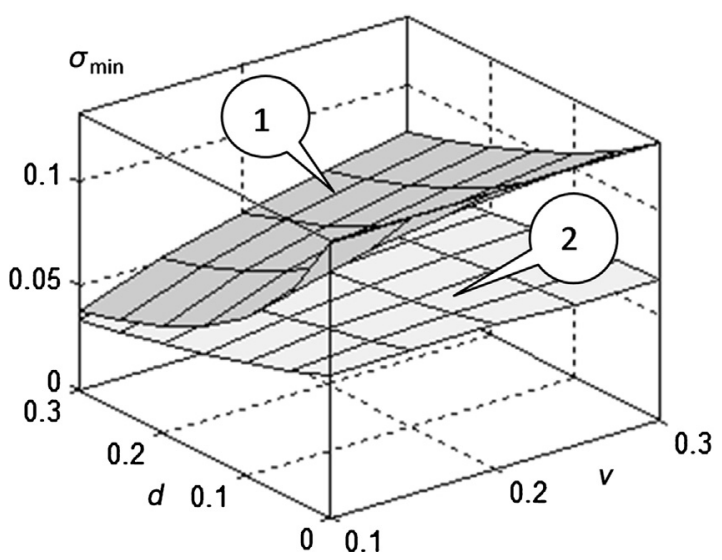

Fig. 5. Minimal reachable $\sigma$ at various $v$ and $d$ for one-time load of a key component (1) and optimized time-distributed load (2) for $n=20$ and $m=5$. 


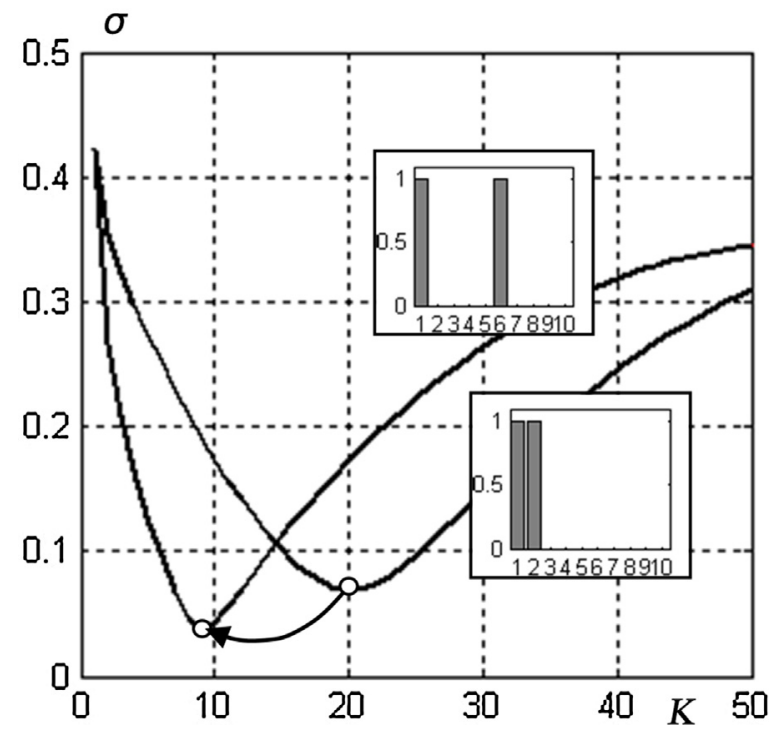

Fig. 6. Influence of the initial vector structure on mixing kinetics.

field. Initially, the key component occupies the two upper cells, and then separates into two parts, one being placed in the top cell, the other in the middle of the mixer. It can be seen from the graphs that the structured load attains $\sigma_{\min }$ twice as fast, and that the value of $\sigma_{\min }$ itself is about half the size. Thus, it can be concluded that the structured load is worthy of attention in mixing technology and mixer design.

\section{Optimal control of particle flows inside a mixer}

It can be easily shown that the matrix of transition probabilities provides an asymptotically homogeneous distribution if

$\sum_{i=1}^{m} P_{i j}=1, \quad i=1, \ldots, m$

i.e., the sum of the entries in each row is equal to one. The matrix given by Eq. (2) meets this condition only if $v=0$ when there is no segregation. The homogeneous distribution becomes unreachable if $v \neq 0$, and we can speak only about when, or how, $\sigma_{\text {min }}$ is reached. However, modifying the particle flows inside the mixer can improve the situation.

Let us make the following simple (and seemingly artificial) transformation in the last column of the matrix

$$
\mathbf{P}=\left[\begin{array}{ccccc}
1-v-d & d & 0 & 0 & v \\
d+v & 1-v-2 d & d & 0 & 0 \\
0 & d+v & 1-v-2 d & d & 0 \\
0 & 0 & d+v & 1-v-2 d & d \\
0 & 0 & 0 & d+v & 1-d-v
\end{array}\right] .
$$

Condition (10) is now automatically satisfied, and the homogeneous asymptotic distribution becomes possible, even in the presence of segregation. The matrix transformation given by Eq. (11) means that the part $v$ of the segregating component must be taken from the bottom cell and moved to the top one. If segregation results from differences in component particle size, this can be realized in practice. It is sufficient to place a sieve near the bottom to separate small particles, and direct these particles to the top by an elevator. This is called mixing with internal circulation of the segregating component.

In the non-linear model described by the matrix in Eq. (4), the values of $v$ are unknown in advance, and numerical experiments

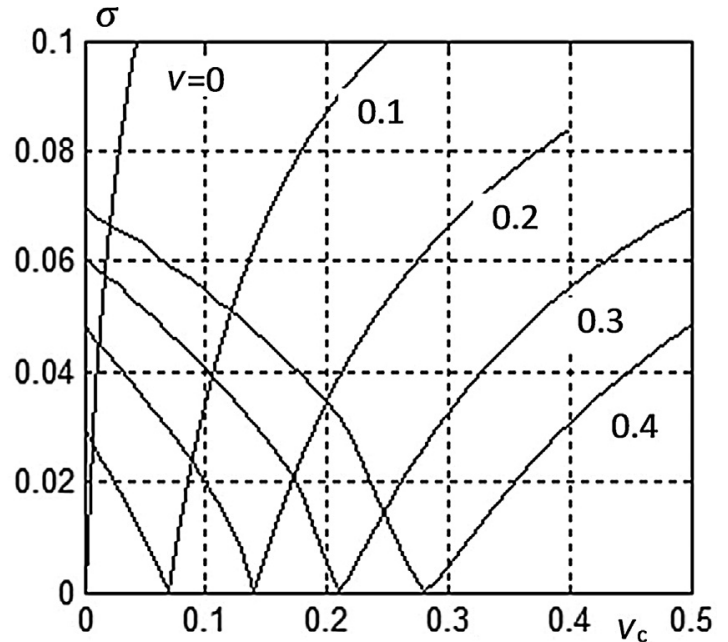

Fig. 7. Influence of circulation rate on $\sigma$ at various $v$ and $d=0.2$.

are required to determine the optimal value of the circulation rate $v_{c}$. An example is shown in Fig. 7 for the value of $\sigma$ versus the circulation rate $v_{c}$ at different segregation rates. The optimal values of $v_{c}$ providing asymptotically homogeneous distributions are lower than the corresponding segregation rates. It was also found that the value of $d$ influences the curve of $\sigma$ versus $v_{c}$, but has no influence on the optimal value $v_{c}$. Thus, the circulation of the segregating component in a mixer allows the development of an asymptotically homogeneous mixture that would be impossible in principle without circulation.

\section{Conclusions}

The theory of Markov chains is well suited to the mixing process so that the matrix of transition probabilities can be considered as a mathematical image of a mixer, with the state and feed vectors representing the current state of the mixture and the feed process to the mixer. This allows some formal optimization problems to be described that are directly related to mixing practice. Several optimal solutions were obtained for the time-distributed load of segregating components to a batch mixer, one-time structured load to a batch mixer, and mixing with internal circulation of segregating components. All these solutions can be more or less easily implemented by new mixer designs, or by reconstructing existing ones. Although there is no detailed experimental validation of the results obtained, the concept of time-distributed loading was implemented in an industrial-scale batch blade mixer with a volume of $300 \mathrm{~L}$ and a vertical shaft, and equipped with a screw feeder for pigment on its upper cover. The components to be mixed were sand, ground PVC waste, and fine-ground heavy pigment, with a strong tendency for segregation. The reconstruction of the mixer enabled a reduction in the pigment $\sigma$ value from 0.04 to 0.015 under the same mixing time. Future developments of this approach will examine the transition to 2D and 3D chain models to account for more features of local particle migration, as well as applications to continuous mixing.

\section{Acknowledgement}

This work is supported by the Russian Foundation for Basic Research (project 15-08-01684).

\section{References}

Ammarcha, C., Gatumel, C., Dirion, J. L., Cabassud, M., Mizonov, V., \& Berthiaux, H. (2013). Transitory powder flow dynamics during emptying of a continuous mixer. Chemical Engineering and Processing, 65, 68-75. 
Berthiaux, H., \& Mizonov, V. (2004). Applications of Markov chains in particulate process engineering: A Review. Canadian Journal of Chemical Engineering, 85 , 1143-1168.

Berthiaux, H., Mizonov, V., \& Zhukov, V. (2005). Application of the theory of Markov chains to model different processes in particle technology. Powder Technology, $157,128-137$

Berthiaux, H., Marikh, K., Mizonov, V., Ponomarev, D., \& Barantzeva, E. (2004). Modelling continuous powder mixing by means of the theory of Markov chains. Particulate Science and Technology, 22, 379-389.

Bridgwater, J. (2010). Mixing of particles and powders: Where next? Particuology, 8 , 563-567.

Bridgwater, J. (2012). Mixing of powders and granular materials by mechanical means-A perspective. Particuology, 10, 397-427.

Danckwerts, P. V.(1953). Continuous flow systems: Distribution of residence times. Chemical Engineering Science, 2, 1-11.

Fan, L. T., Lai, F. S., Akao, Y., Shinoda, K., \& Yoshizawa, E. (1978). Numerical and experimental simulation studies on the mixing of particulate solids and the synthesis of a mixing system: Mixing process and stochastic motion of mutually noninteracting particles. Computers \& Chemical Engineering, 2(1), 19-32.

Iddir, H., Arastoopour, H., \& Hrenya, C. M. (2005). Analysis of binary and ternary granular mixture behavior using the kinetic theory approach. Powder Technology, 151, 117-125.

Marikh, K., Berthiaux, H., Mizonov, V., Barantseva, E., \& Ponomarev, D. (2006). Flow analysis and Markov chain modelling to quantify the agitation effect in a continuous mixer. Chemical Engineering Research and Design, 84(11), 1059-1074.

Sommer, K. (1996). Mixing of particulate solids. KONA Powder and Particle Journal, $14,73-78$.

Wang, R. H., \& Fan, L. T. (1976). Axial mixing of grains in a motionless Sulzer (Koch) mixer. Industrial \& Engineering Chemistry Process Design and Development, 15, 381-388.

Wang, R. H., \& Fan, L. T. (1977). Stochastic modeling of segregation in a motionless mixer. Chemical Engineering Science, 32, 695-701. 